\title{
Magnetic resonance image-based 3D volume interstitial brachytherapy using polyether ether ketone catheters in advanced cervical cancer - a feasibility study
}

\author{
Siddanna Rudrappa Palled, MD, DNB, Chitradurga Abdul Razak Tanvirpasha, MD, DNB, Thimmaiah Naveen, MD, \\ Saminathan Sathiyan, PhD, Ganesh Kadirampatti, PhD, Vishwanath Lokesh, MD \\ Department of Radiation Oncology, Kidwai Memorial Institute of Oncology, Bangalore, India
}

\begin{abstract}
Purpose: A feasibility study of 3D volume-based interstitial brachytherapy using thermoplastic polyether ether ketone (PEEK) catheters in advanced cervical cancer MR-based brachytherapy, when MR-compatible applicators are not available.

Material and methods: Five cervical cancer patients received EBRT dose of 50 Gy in 25 fractions to whole pelvis using 3D conformal radiotherapy after standard pre-treatment evaluation. In our institute, interstitial brachytherapy (ISBT) is planned in BrachyVision TPS and routinely performed using stainless steel needles with Syed-Neblett template. Standard MR-compatible ISBT applicators were not available; therefore, the implant was carried out using PEEK catheters and Syed-Neblett template. The T1 and T2 MR and CT images of patients were acquired for treatment planning. Gross tumor volume (GTV), high-risk clinical target volume (HR-CTV), intermediate-risk clinical target volume (IR-CTV), and organs at risk (OARs) were delineated with the use of MR T2 weighted images, following GEC-ESTRO guidelines. Volumetric optimization was used for planning, and the treatment plan was executed with high-dose-rate (HDR) ${ }^{192}$ Ir Gammamed Plus device.

Results: The dose prescribed to HR-CTV was 30 Gy low-dose-rate (LDR) equivalent (5 Gy $\times 4$ fractions). Doses to target volume and OARs were analyzed using dose volume histogram (DVH). Dose values were biologically normalized to equivalent doses of $2 \mathrm{~Gy}$ fractions $\left(\mathrm{EQD}_{2}\right)$. The average tumor volume was $67 \mathrm{cc}$ and the average $\mathrm{D}_{90} \mathrm{Of} \mathrm{HR}-\mathrm{CTV}$


of rectum and bladder were $70 \mathrm{~Gy}$ and $76 \mathrm{~Gy} \mathrm{EQD}_{2}$ (3.71 Gy and $4.04 \mathrm{~Gy}$ dose/fraction), respectively.

Conclusions: When the facilities for MR-compatible applicators are not available, MR-based ISBT is feasible with PEEK catheters using available resources for advanced cervical cancer. Doses to HR-CTV and IR-CTV were achieved, restricting doses to OARs as per GEC-ESTRO guidelines.
\end{abstract}

Key words: MR imaging, cervical cancer, interstitial brachytherapy.

\section{Purpose}

Cervical cancer is a common cancer in women in India and more than $2 / 3$ of patients present at an advanced stage. Recent data of the National Cancer Registry Programme (NCRP) shows that the incidence accounts for $15.7 \%$ of all cancers [1,2]. Radical radio-chemotherapy, including external beam followed by brachytherapy (BT), is a standard of care. Brachytherapy forms the cornerstone for local control rates and toxicities [3,4]. Brachytherapy is generally applied as high-dose-rate (HDR) fractionated treatment delivered by intracavi- tary (IC) alone, or IC and interstitial or interstitial alone. Brachytherapy offers the advantage of delivering a very high-dose to the tumor and low doses to adjacent organs at risk (OARs).

Recently, a paradigm shift in BT practice from conventional planning to image-based brachy approach was implemented. Image-based target volumes related to disease at the time of BT have been published by GECESTRO $[5,6]$. Tumor volume is well recognized as one of the most important prognostic factors in terms of local control. Magnetic resonance (MR) imaging-based definitions are the gold standard and have shown promising 
results in many mono-institutional series and a multicenter study [7]. MR-based BT approach mandates the use of MR-compatible applicators, MR imaging, and adaptive planning to achieve optimal doses to target and OARs sparing. There is an ongoing research in terms of variety of applicators and optimization techniques to achieve better local control and sparing of OARs. Han et al. has shown better sparing of OARs with the use of novel direction modulated brachytherapy (DMBT) tandem applicator in small volume disease [8].

This feasibility study evaluates the use of plastic polyether ether ketone (PEEK) catheters to initiate MR-guided BT and report the preliminary experience of utilizing plastic catheters for MR image-guided BT approach for advanced cervical cancer.

\section{Material and methods}

This study includes locally advanced and post-operative cervical cancer patients. As a pilot study, five patients were included and among them, three were FIGO stage IIIB, one post-operative, and one post-operative recurrent case. All these patients underwent standard pre-treatment evaluation of complete history, physical examination, and examination under anesthesia (EUA). Cystoscopy, proctoscopy, routine hematological, and renal function tests were performed. Imaging included chest radiography, computed tomography (CT) scans of abdomen and pelvis, and bone scan if clinically indicated. Patients received external beam radiotherapy dose of $50 \mathrm{~Gy}$ in 25 fractions to whole pelvis using 3D conformal therapy. If the gross tumor volume (GTV) at the time of brachytherapy was more than $4 \mathrm{~cm}$ with the parametrial extension, interstitial brachytherapy was performed using PEEK catheters with Syed-Neblett template. CT scan images were acquired with $3 \mathrm{~mm}$ axial slices and treatment planning was performed using BrachyVision TPS (Varian Medical Systems, Palo Alto, CA, USA) with volume optimization.

Recently, in our center, 1.5 Tesla MRI device was implemented into the clinical use. However, MR-compatible brachytherapy applicators were not available; therefore, the implant procedure was performed with semi-flexi-

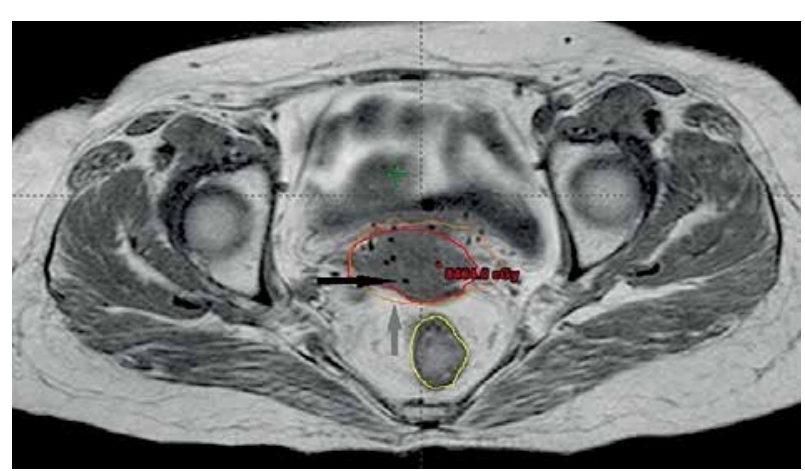

Fig. 1. T2 MR image axial view, black arrow showing catheters and grey arrow indicates HR-CTV covered with 5 Gy isodose line ble thermoplastic PEEK catheters and Syed-Neblett template. This plastic catheter usage is prevalent in prostate and breast brachytherapy; the catheters are biocompatible and the whole applicator set is MR-compatible. The thermoplastic PEEK catheters used in our study were manufactured in India.

$\mathrm{T} 1$ and T2 relaxation MR images were acquired for volume delineation and planning; CT images were also obtained to visualize the catheters' position by placing copper dummies in the catheters. The DICOM (digital imaging and communication in medicine) images of MRI and CT were imported into BrachyVision TPS for planning.

Gross tumor volume, high-risk clinical target volume (HR-CTV), intermediate-risk clinical target volume (IR-CTV), and OARs were delineated with the use of MR T2 weighted images, following GEC-ESTRO guidelines. HR-CTV delineation was challenging, as prebrachy MR images were not available. This was overcome by changing the contrast resolution and EUA findings (Figure 1). T1 weighted images were helpful for a catheter reconstruction, and the catheters were better appreciated as black straight lines without any interruptions. Haack et al. showed that plastic catheters are well suited for MR-based reconstruction in T1 images and all catheters were visible without artifacts [9]. Depending of the extent of disease, the active lengths of the implant ranged from $6-8 \mathrm{~cm}$. The dose prescribed was $20 \mathrm{~Gy}$ in 4 fractions (30 Gy low-dose-rate [LDR] equivalent $\left[\mathrm{EQD}_{2}\right]$ ) to HR-CTV, 6 hours apart, and delivered over two days. Doses of 2 cc of bladder and rectum were restricted to less than 4.0 and 3.7 Gy per fraction, respectively. With our initial experience, the dose escalation was considered to be 6 Gy per fraction. Treatment plans were performed using volumetric optimization with $5 \mathrm{~mm}$ dwell position (Figure 2). The OARs constraints were met using manual dragging of isodose lines. The treatment was executed using ${ }^{192} \mathrm{Ir}$ Gammamed Plus HDR remote afterloading machine (Varian Medical Systems, Palo Alto, CA, USA), with ${ }^{192} \mathrm{Ir}$ high activity of $10 \mathrm{Ci} \max$, source of $4.6 \mathrm{~mm}$ active length and $0.9 \mathrm{~mm}$ in diameter.

\section{Results}

The prescribed dose to HR-CTV was 5 Gy $\times 4$ fractions (30 Gy LDR equivalent). The dose to HR-CTV and OARs were analyzed using DVH. Dose values were biologically normalized to equivalent doses of $2 \mathrm{~Gy}$ fractions $\left(\mathrm{EQD}_{2}\right.$, equivalent to $50 \mathrm{cGy} / \mathrm{h} \mathrm{LDR}$, using spreadsheet of the Medical University in Vienna) by applying the linear-quadratic model. The 3D dose-volume parameters for five patients are shown in Table 1 . The average tumor volume was $67 \mathrm{cc}$, the average dose to $90 \%$ of HR-CTV $\left(\mathrm{D}_{90}\right)$ volume was $5.7 \mathrm{~Gy}(79 \mathrm{~Gy} \mathrm{EQD})_{2}$, and the average dose to $100 \%$ of HR-CTV $\left(\mathrm{D}_{100}\right)$ volume was $3.8 \mathrm{~Gy}$ (68 Gy $\mathrm{EQD}_{2}$ ). The average doses of $2 \mathrm{cc}$ of rectum and bladder were 3.71 Gy and $4.04 \mathrm{~Gy}$, dose maximum were $70 \mathrm{~Gy}$ and $76 \mathrm{~Gy} \mathrm{EQD}_{2}$, respectively. High-dose volumes $\mathrm{V}_{150}$ and $\mathrm{V}_{200}$ were $28 \mathrm{cc}$ and $14 \mathrm{cc}$, respectively, with close proximity to the catheters. 


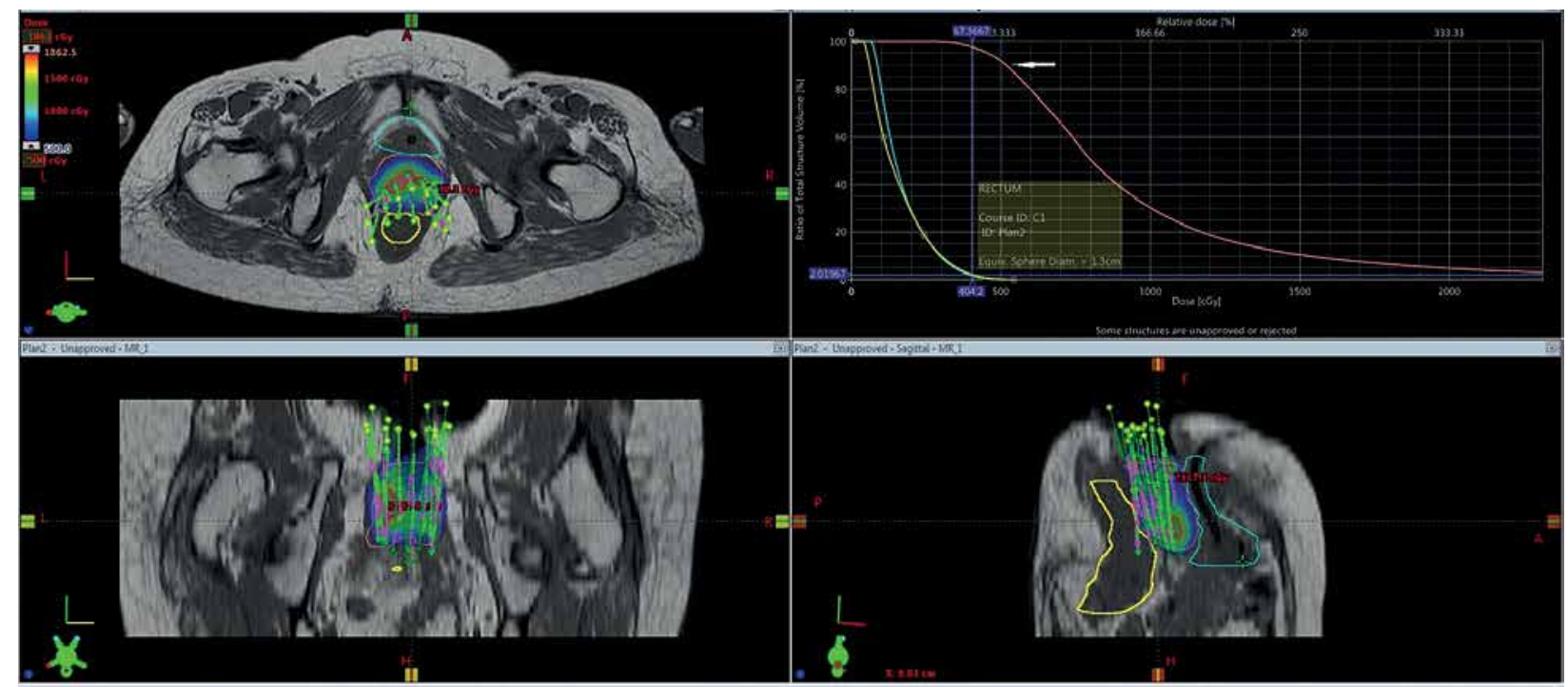

Fig. 2. Axial, coronal and sagittal view of isodose distribution with DVH on MR images (white arrow represents $\mathrm{D}_{90}$ in DVH)

Table 1. Dose-volume parameters for HR-CTV and organs at risk (OARs)

\begin{tabular}{|c|c|c|c|c|c|c|c|c|c|c|}
\hline $\begin{array}{l}\text { HDR } \\
\text { (Gy/\#) }\end{array}$ & $\begin{array}{c}\mathrm{EQD}_{2} \\
\text { (2 Gy } \\
\text { equiva- } \\
\text { lent) }\end{array}$ & $\begin{array}{c}\text { Tumor } \\
\text { volume } \\
\text { in cc }\end{array}$ & $\begin{array}{c}\mathrm{D}_{90} \mathrm{cGY} \\
\left(\mathrm{EQD}_{2}\right)\end{array}$ & $\begin{array}{c}\mathrm{D}_{100} \mathrm{cGY} \\
\left(\mathrm{EQD}_{2}\right)\end{array}$ & $\mathrm{V}_{150}[\mathrm{cc}]$ & $\begin{array}{l}V_{200} \\
{[\mathrm{cc}]}\end{array}$ & $\begin{array}{l}\text { Rectum } 2 \\
\text { cc dose }\end{array}$ & $\begin{array}{c}\text { Total } 2 \\
\text { cc rectal } \\
\text { dose } \\
\text { (HDR } \\
\text { dose) }\end{array}$ & $\begin{array}{l}\text { Bladder } \\
2 \text { cc dose }\end{array}$ & $\begin{array}{c}\text { Total } 2 \text { cc } \\
\text { bladder } \\
\text { dose } \\
\text { (HDR } \\
\text { dose) }\end{array}$ \\
\hline $5 \times 4$ & 25 & 55 & 594 & 378 & 37 & 22 & 346 & (17) 67 & 413 & (23) 73 \\
\hline $5 \times 4$ & 25 & 62 & 496 & 359 & 29 & 12 & 314 & (16) 66 & 342 & (17) 67 \\
\hline $5 \times 4$ & 25 & 89 & 488 & 325 & 17 & 8 & 359 & (19) 69 & 364 & 69 (19) \\
\hline $\begin{array}{l}6 \times 3 \\
5 \times 1\end{array}$ & 28 & 62 & 690 & 522 & 30 & 14 & 483 & (28) 78 & 527 & 83 (33) \\
\hline $\begin{array}{l}6 \times 3 \\
5 \times 1\end{array}$ & 30 & 65 & 516 & 317 & 25 & 12 & 355 & (18) 68 & 375 & (20) 70 \\
\hline AVG & 26.5 & 67 & $557 / 29$ & $380 / 18$ & 28 & 14 & 371 & (20) 70 & 404 & (26) 76 \\
\hline
\end{tabular}

$H D R$ - high-dose-rate, AVG - average, \#-fraction, cc-cubic centimeter

\section{Discussion}

Image-guided brachytherapy is the standard of care, either in the form of orthogonal X-rays for 2D or CT imaging for the 3D volume-based brachytherapy. The volumetric imaging is superior than the planar imaging in terms of doses to tumor and OARs [10,11]. Tumor volume coverage with an adequate dose is important prognostic factor for better local control rates. Standard intracavitary brachytherapy does not cover the tumor volume adequately in locally advanced disease and in distorted anatomy [12]. Syed et al. developed a technique of interstitial/intracavitary brachytherapy to improve the dose distribution in patients with locally advanced disease [13], and along with Aristizabal et al. have reported good results using similar techniques [14,15].

Routinely at our center, interstitial brachytherapy (ISBT) is applied for locally advanced cancer cervix patients using Syed-Neblett template with stainless steel needles, and treatment volume is planned based on EUA findings. Initially, the treatment plans were performed on X-ray planar images and later on moved to CT images.

Dimopoulos et al. published the utility of MR imaging in tumor delineation in cancer cervix. The GECESTRO Working Group (IV) guidelines stated the basic principles and parameters for MR multi-planar image in image-based adaptive brachytherapy for cancer cervix [16]. Vienna group of Pötter et al. [7] has developed ring applicator for intracavitary brachytherapy. Learning form failures in controlling the parametrial disease, the Vienna group modified the ring applicator to Vienna II applicator, with interstitial facility to adequately cover the HR-CTV. They demonstrated that the image-guided adaptive brachytherapy (IGABT) using Vienna II intracavitary/interstitial (IC/IS) applicator has led to a significant improvement of local control in locally advanced cervical cancer (LACC) patients, when 
comparing to previous results. Derks et al. in a retrospective study has shown the effect of 2D conventional brachytherapy (CBT) compared to 3D MRI-guided brachytherapy (IGBT) with and without the use of interstitial needles, showing an improved local control and reduced toxicities [17].

The GYN GEC-ESTRO Working Group guidelines (I and II) focus on 3D dose-volume parameters for brachytherapy of cervical carcinoma. They recommend $\mathrm{D}_{90}, \mathrm{D}_{100}$, and dose of 2 cc to OARs [5,6].

Many oncologic centers lack the facility for MR image-guided brachytherapy. Since our center has the 1.5 Tesla MR, we are in the process of acquiring MR-compatible intracavitary/interstitial (IC/IS) brachytherapy applicators. To overcome the lack of facility, a new technique was implemented using Syed-Neblett template with PEEK catheters for interstitial implantation. The whole ISBT set was made MR-compatible. These catheters are firm with a sharp tip and are inserted using a stainless-steel stylet that facilitates the catheter to pass through the tumor and minimizes the convergence of catheters at cranial end.

In the first patient, the $\mathrm{CT}$ planning was performed using copper dummies to delineate the catheters. The dose-volume optimization was done in CT images, and later on MR image sequences that were acquired for planning. The identification of catheters and catheter tracking was not difficult, but the delineation of GTV was problematic due to implant geometry and contrast resolution as well as pre-treatment MR images not available. The HR-CTV delineation was completed using contrast resolution in T2 sequences and findings from clinical examination under anesthesia and to some extent, with initial CT findings. The patient comfort with plastic catheter was excellent in terms of pain.

In the initial three cases, the prescription dose was 20 Gy in 4 fractions to the HR-CTV, which was $30 \mathrm{~Gy}$ LDR equivalent, as this being the institute's practice. The average HR-CTV $\left(\mathrm{D}_{90}\right)$ was $67 \mathrm{~cm}^{3}$ and was adequately covered with an average dose of $5.57 \mathrm{~Gy}$; dose to $100 \%$ HR-CTV $\left(D_{100}\right)$ was 3.8 Gy per fraction. As per GECESTRO recommendations, 2 cc of bladder and rectal doses were $3.71 \mathrm{~Gy}$ and $4.04 \mathrm{~Gy}$, respectively, which were $70 \%$ and $85 \%$ of the prescribed dose, respectively. The total doses of $2 \mathrm{cc}$ of rectum and bladder were 70 and 76 Gy, respectively (Table 1). With our initial experience, the dose escalation was considered to be $6 \mathrm{~Gy} /$ fraction for 3 fractions. High-dose volumes, $V_{150}$ and $V_{200}$, were $28 \mathrm{cc}$ and $14 \mathrm{cc}$, respectively, with close proximity to the catheters. As this is our initial experience with PEEK catheters used for interstitial implant, the dose to HR-CTV was restricted to $80 \mathrm{~Gy} \mathrm{EQD}_{2}$. With long-term follow-up and depending on the toxicity profiles, further dose escalation to HR-CTV could be considered.

\section{Conclusions}

When the facilities for MR-compatible applicators are not available, MR-based interstitial brachytherapy is feasible with the PEEK catheters. Patient comfort was excel- lent with respect to pain. Further dose escalation could be considered based on toxicity profiles.

\section{Disclosure}

Authors report no conflict of interest.

\section{References}

1. Nandakumar A, Ramnath T, Chaturvedi M. The magnitude of cancer cervix in India. Indian J Med Res 2009; 130: 219-221.

2. Shah B, Nandakumar A, Shukla DK et al. National Centre for Disease Informatics Research. National Cancer Registry Programme. ICMR. Three year report of population based registries 2009-2011. Bangalore, India: NCDIR-NCRP (ICMR) 2013.

3. Lanciano RM, Minhee W, Lawrence RC et al. Pretreatment and treatment factors associated with improved outcome in squamous cell carcinoma of the uterine cervix: a final report of the 1973 and 1978 patterns of care studies. Int J Radiat Oncol Biol Phys 1991; 20: 667-676.

4. Montana GS, Hanlon MS, Brickner TJ et al. Carcinoma of the cervix: patterns of care studies: review of 1978, 1983, and 1988-1989 surveys. Int J Radiat Oncol Biol Phys 1995; 32: 1481-1486.

5. Haie-Meder C, Pötter R, Limbergen EV et al. Recommendations from Gynaecological (GYN) GEC-ESTRO Working Group (I): concepts and terms in 3D image based 3D treatment planning in cervix cancer brachytherapy with emphasis on MRI assessment of GTV and CTV. Radiother Oncol 2005; 74: 235-245.

6. Pötter R, Haie-Meder C, Limbergen EV et al. Recommendations from gynaecological (GYN) GEC ESTRO working group (II): concepts and terms in 3D image-based treatment planning in cervix cancer brachytherapy-3D dose volume parameters and aspects of 3D image-based anatomy, radiation physics, radiobiology. Radiother Oncol 2006; 78: 67-77.

7. Pötter R, Georg P, Dimopoulos J et al. Clinical outcome of protocol based image (MRI) guided adaptive brachytherapy combined with 3D conformal radiotherapy with or without chemotherapy in patients with locally advanced cervical cancer. Radiother Oncol 2011; 100: 116-123.

8. Han DY, Safigholi H, Soliman A et al. Direction modulated brachytherapy for treatment of cervical cancer. II: Comparative planning study with intracavitary and intracavitary-interstitial techniques. Int J Radiat Oncol Biol Phys 2016; 96: 440-448.

9. Haack S, Nielsen SK, Lindegaard JC et al. Applicator reconstruction in MRI 3D image-based dose planning of brachytherapy for cervical cancer. Radiother Oncol 2009; 91: 187-193.

10. Datta NR, Srivastava A, Maria Das KJ et al. Comparative assessment of doses to tumor, rectum, and bladder as evaluated by orthogonal radiographs vs. computer enhanced computed tomography-based intracavitary brachytherapy in cervical cancer. Brachytherapy 2006; 5: 223-229.

11. Jamema SV, Saju S, Mahantshetty U et al. Dosimetric evaluation of rectum and bladder using image-based CT planning and orthogonal radiographs with ICRU 38 recommendations in intracavitary brachytherapy. J Med Phys 2008; 33: 3-8.

12. Chakrabarti B, Pal SK, Sepai HM et al. Clinical and dosimetric consequences of imperfect applicator insertion in cervical cancer brachytherapy. J Contemp Brachytherapy 2018; 10: 321-336. 
13. Syed AN, Puthawala AA, Neblett D et al. Transperineal interstitial-intracavitary "Syed-Neblett" application in the treatment of carcinoma of the uterine cervix. Endocurie Hypertherm Oncol 1986; 2: 1-13.

14. Syed AN, Puthawala AA, Abdelaziz NN et al. Long-term results of low-dose-rate Interstitial-intracavitary brachytherapy in the treatment of carcinoma of the cervix. Int J Radiat Oncol Biol Phys 2002; 54: 67-78.

15. Aristizabal SA, Woolfitt B, Valencia A et al. Interstitial parametrial implants in carcinoma of the cervix stage IIB. Int J Radiat Oncol Biol Phys 1986; 13: 445-450.

16. Dimopoulos J, Petrow P, Tanderup K et al. Recommendations from Gynecological (GYN) GEC-ESTRO Working Group (IV):Basic principles and parameters for MR imaging within the frame of image based adaptive cervix cancer brachytherapy. Radiother Oncol 2012; 103: 113-122.

17. Derks K, Steenhuijsen JLG, van den Berg HA et al. Impact of brachytherapy technique (2D versus $3 \mathrm{D}$ ) on outcome following radiotherapy of cervical cancer. J Contemp Brachytherapy 2018; 10: 17-25. 